\title{
Ameboma del recto con absceso hepático asociado: reporte de un caso
}

\section{Case Report of Rectal Ameboma with Associated Liver Abscess}

Faruk Esteban Erebrie G., MD, ${ }^{1}$ William Hernán Valencia G., MD, ${ }^{2}$ Luis Gonzalo Guevara C., MD, ${ }^{3}$ René Marcelo Escobar P., MD. ${ }^{4}$

\footnotetext{
Especialista en Gastroenterología Clínica, Hospital Universitario San Vicente Fundación. Medellín, Colombia.

Correo electrónico: farukerebrie@hotmail.com

2 Especialista en Gastroenterología Clínica, Hospital Universitario San Vicente Fundación. Medellín, Colombia.

Correo electrónico: wihvalen@yahoo.com

${ }^{3}$ Especialista en Hepatología. Especialista en Gastroenterología Clínica. Hospital Universitario San Vicente Fundación. Medellín, Colombia.

Correo electrónico: gonzogastro@gmail.com

${ }^{4}$ Especialista en Cirugía Gastrointestinal y Endoscopia

Digestiva. Hospital Universitario San Vicente

Fundación. Medellín, Colombia.

Correo electrónico: remar_med@hotmail.com
}

Fecha recibido: $28-05-14$

Fecha aceptado: 02-02-15

\begin{abstract}
Resumen
La infección por Entamoeba histolytica sigue siendo un problema mayor de salud pública en los países en desarrollo como Colombia. Aunque en la gran mayoría de casos se presenta como una disentería o un absceso hepático amebiano, existen casos donde la evolución genera una verdadera masa inflamatoria en el colon llamada ameboma. Estos amebomas son fácilmente confundidos con una lesión neoplásica y, de no tener un gran nivel de sospecha clínica o endoscópica, terminan en una resección de colon con un diagnóstico posoperatorio dado por la presencia de trofozoítos sin malignidad. Sin embargo, cuando el diagnóstico se hace de manera preoperatoria, podría haber resolución del cuadro sin necesidad de cirugía. Exponemos el caso de una rara presentación de ameboma en el recto asociado con un absceso hepático perforado, en el cual se hizo un diagnóstico preoperatorio, pero dada la ruptura de la lesión en el hígado, requirió de una intervención quirúrgica.
\end{abstract}

\section{Palabras clave \\ Entamoeba histolytica, ameboma, absceso hepático amebiano.}

\begin{abstract}
Entamoeba histolytica infections continue to be a major public health problem in developing countries such as Colombia. Although it most often presents as dysentery or amebic liver abscess, there are cases in which a true inflammatory mass develops in the colon. This is called an ameboma. These amebomas are easily confused with neoplastic lesions so that in the absence of a high level of clinical and endoscopic suspicion, a colon resection may be performed with the postoperative diagnosis given by the presence of trophozoites without malignancy. However, when the diagnosis is made preoperatively symptoms can be without surgery. We report a rare presentation of ameboma in the rectum associated with a perforated liver abscess. This was diagnosed prior to surgey, but given the liver injury, required surgery.
\end{abstract}

\section{Keywords}

Entamoeba histolytica, ameboma, amebic liver abscess.

\section{INTRODUCCIÓN}

La amebiasis causada por Entamoeba histolytica es un problema de salud con marcada incidencia en países en desarrollo. El cuadro clínico se mueve en el espectro del paciente asintomático, el cuadro de colitis con diarrea disentérica, el absceso hepático y la infección crónica localizada de la pared del colon que forma una verdadera masa llamada ameboma o granuloma amebiano. Esta última forma de presentación es bastante infrecuente, siendo el ciego y el colon derecho los sitios donde se presenta de manera más común. La presentación mixta con ameboma y absceso 
hepático también ha sido vista en algunos casos e incluso puede ser fuente de confusión con un carcinoma de colon metastásico.

\section{DESCRIPCIÓN DEL CASO CLÍNICO}

Paciente masculino de 55 años de edad, procedente de Medellín, Antioquia, obrero, con historia de tabaquismo (8 cigarrillos/día durante 15 años) y estreñimiento crónico de 8 años de evolución con episodios de rectorragia frecuentes $\sin$ dolor abdominal ni anal, asociados con una pérdida de 8 kilogramos de peso en todo ese tiempo. En noviembre de 2011, le realizan rectosigmoidoscopia que arroja el diagnóstico de proctitis ulcerativa moderada; en las biopsias se reporta colitis linfocítica, para la cual inicia tratamiento con sulfasalazina $1 \mathrm{~g} /$ día, mesalazina enemas, dos veces al día, y ácido fólico. Una nueva colonoscopia, llevada a cabo en octubre de 2012, mostró pancolitis ulcerativa activa pero contradictoriamente las biopsias son reportadas como "pólipo hiperplásico". Aun así, se inicia el manejo con mesalazina $4 \mathrm{~g}$ /día, prednisona 40 $\mathrm{mg} /$ día y ácido fólico con la estrategia de valorar la respuesta clínica y endoscópica a los 3 meses.

Ingresa al Hospital Universitario San Vicente de Paúl el 08-01-2013 por aumento del número de deposiciones y del sangrado, así como mayor pérdida de peso. Al examen físico se encontró desnutrido, con palidez mucocutánea, hidratado, con signos vitales normales y abdomen no doloroso. Estudios iniciales: leucocitosis de $13600, \mathrm{PMN} 80 \%$, $\mathrm{Hb}$ 12,6 gm/dL, plaquetas 360 000, química sanguínea, perfil hepático y tiempos de coagulación normales, albúmina $2,5 \mathrm{mg} / \mathrm{dL}$. Coprológico reportado sin parásitos. La colonoscopia realizada el 11-01-2013 (figura 1) muestra mucosa del recto con múltiples úlceras de diferentes tamaños que alternan con áreas de mucosa normal y una lesión tumoral circunferencial, necrosada, estenosante, friable y con sangrado fácil, que no es posible franquear y de la cual se toman biopsias para descartar malignidad frente a enfermedad de Crohn. El informe de patología reporta

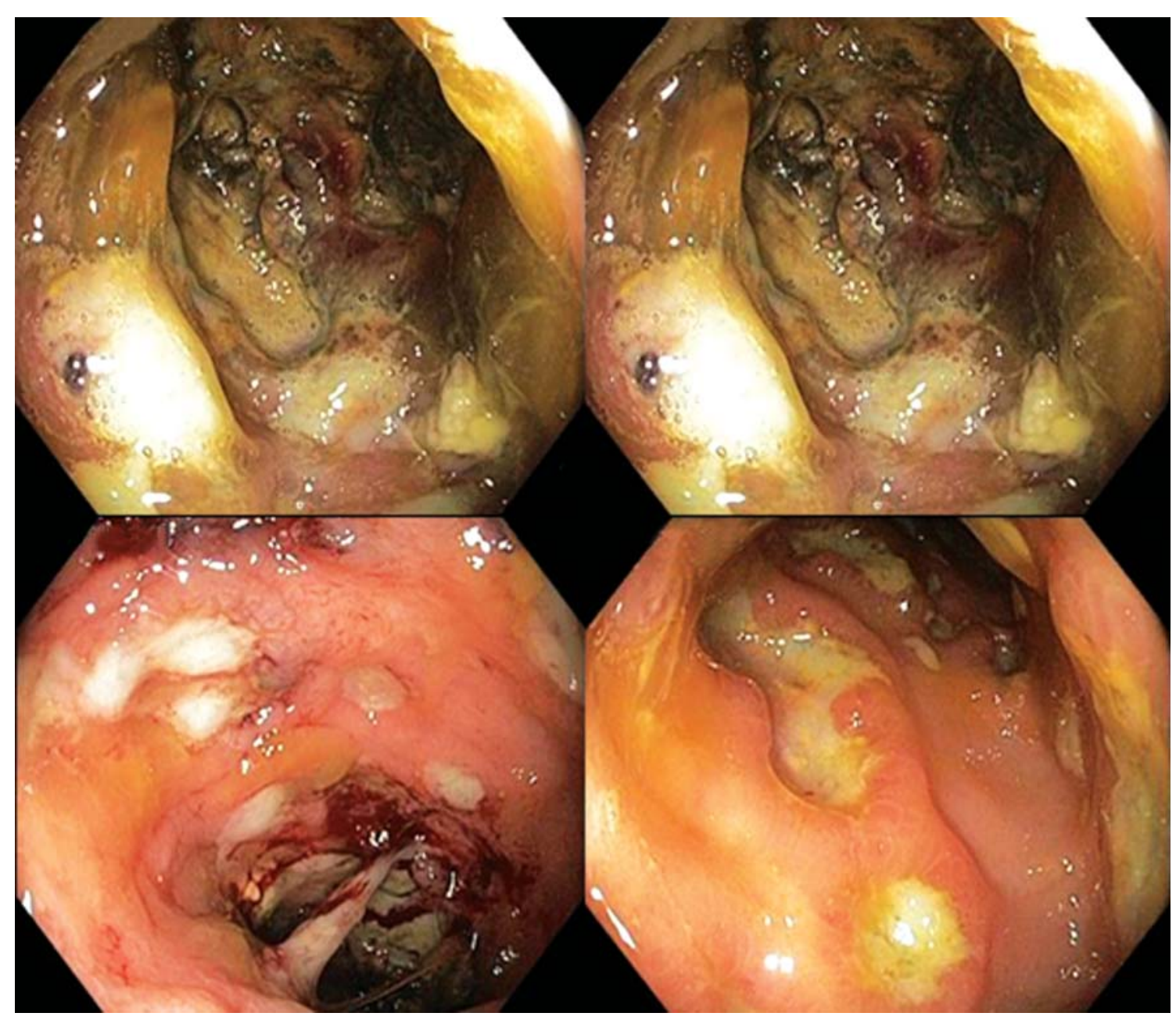

Figura 1. Colonoscopia realizada el 11 de enero de 2013. 
textualmente "fragmentos de mucosa colónica, con denso infiltrado inflamatorio mononuclear a nivel de la lámina propia, necrosis extensa, proliferación neoplásica maligna constituida por células pequeñas a medianas de citoplasma escaso y núcleos redondos hipercromáticos y nucléolos visibles, sin figuras mitóticas. Marcadores de inmunohistoquímica CD3, CD20, CD10, BCL6, BCL2 y ciclina D1, todos negativos. Conclusión: neoplasia maligna en clasificación. Dado que la muestra está constituida por tejido necrótico, no es posible llegar a un diagnóstico concluyente, por lo que se solicitan nuevas biopsias de tejido viable". Una TAC contrastada de abdomen (figura 2) muestra dos lesiones hepáticas compatibles con abscesos y cambios por pancolitis. Los estudios de VIH, ANA, ANCA, serologías de hepatitis viral y tuberculina son negativos. Se inicia manejo con ciprofloxacina y metronidazol IV.

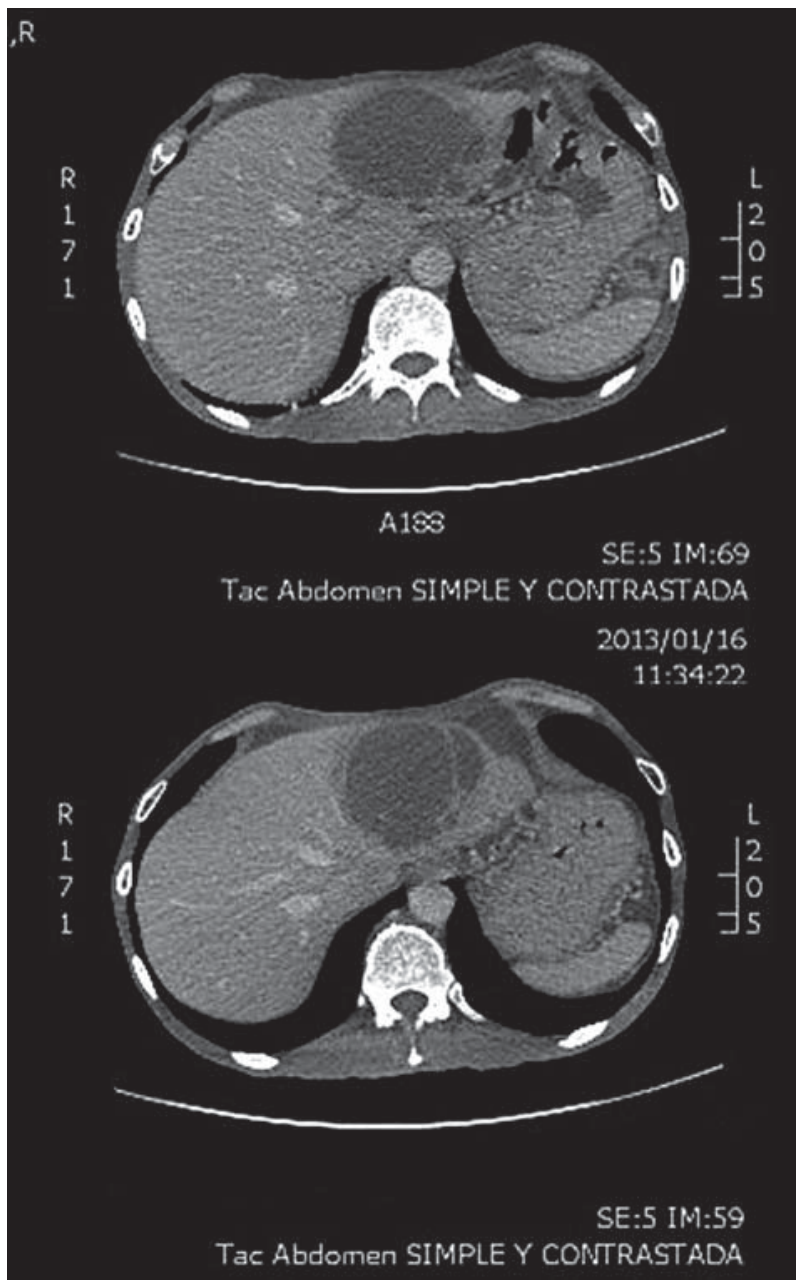

Figura 2. TAC contrastada de abdomen.

En otra colonoscopia llevada a cabo el 18-01-2013, las biopsias reportan nuevamente denso infiltrado inflamatorio mononuclear a nivel de la lámina propia, necrosis extensa, úlcera activa con invasión severa por trofozoítos de ameba y ausencia de malignidad. Se continuó el manejo antibiótico intravenoso. El día 25-01-2013 el paciente refiere dolor abdominal intenso súbito y se encuentran con signos de irritación peritoneal.

Se lleva a laparotomía en la que se observa peritonitis purulenta de cuatro cuadrantes, absceso hepático del lóbulo hepático izquierdo roto, con una cavidad del absceso de \pm 15 $x 10 \mathrm{~cm}$, masa rectal de $4 x 4 \mathrm{~cm}$ con necrosis, perforación y pérdida de la pared rectal que genera un absceso pélvico con materia fecal en su interior. Se encontró que el colon derecho y transverso era de aspecto macroscópico normal, aunque se apreciaron úlceras en la mucosa del colon descendente. Se realiza drenaje de peritonitis y del absceso pélvico, resección del colon descendente, sigmoide y recto proximal, incluida la lesión inflamatoria, cierre del muñón rectal y colostomía tipo Hartmann, lavado peritoneal y cierre de cavidad abdominal. El paciente permaneció 48 horas en la unidad de cuidados intensivos (UCI) y después presentó evolución favorable, con egreso a los 8 días posquirúrgicos.

El informe de patología macroscópica reporta colon con áreas necróticas, membranas fibrinopurulentas y sitio de perforación de $10 \mathrm{~cm}$ de longitud, asociado con la presencia de una masa indurada de $4,5 \mathrm{~cm}$ de diámetro mayor. La descripción microscópica relata extensas úlceras cubiertas por fibrina, abundantes macrófagos, úlceras en botón de camisa, microabscesos, elongación de las criptas, severos cambios nucleares reactivos, trofozoítos de ameba y zona de perforación con necrosis de licuefacción.

Luego se realizó cierre de la colostomía, con previa colonoscopia que mostró colon y muñón rectal con mucosa completamente normal.

\section{DISCUSIÓN}

La amebiasis continúa siendo un problema de salud endémico en Colombia a pesar de la mejoría de las condiciones higiénicas del área urbana y rural de nuestro país (1). Sin embargo, los ataques agudos de colitis amebiana con diarrea disentérica reciben actualmente un mejor y más rápido tratamiento, lo cual disminuye sus complicaciones (2).

El ameboma o granuloma amebiano es una lesión que muy frecuentemente simula un tumor colorrectal (3-5) que resulta de los cambios hipertróficos y de la formación de tejido de granulación de la pared del colon que rodea una gran ulceración a la que se añade una infección bacteriana secundaria y acumulación de colágeno $(6,7)$. Los síntomas suelen ser diarrea recurrente, sangrado digestivo inferior, dolor abdominal inferior, pérdida de peso, fiebre de bajo grado, masa abdominal palpable e incluso obstrucción intestinal $(2,6)$. El ameboma se vuelve sintomático mucho tiempo después de un ataque agudo de disentería que no 
recibió tratamiento o que fue insuficientemente tratado, incluso años después (8).

Usualmente los amebomas son solitarios pero pueden ser múltiples, son más comunes en hombres entre los 20 y los 60 años de edad y los sitios más frecuentes de presentación son el ciego, el apéndice y el colon sigmoide, seguidos por mucho del ángulo hepático, el colon transverso y el ángulo esplénico (9). Se ha estimado que de todos los casos de amebiasis, solo en un $1,5 \%$ hay formación de amebomas $(2,7)$.

Una muestra aislada de materia fecal o un coprológico seriado pueden colaborar con el diagnóstico si se observan trofozoítos o incluso los quistes; sin embargo, estos podrían no encontrarse en pacientes que no tengan el cuadro reciente de disentería. El diagnóstico endoscópico depende notablemente de un altísimo índice de sospecha por parte del endoscopista. Las biopsias tomadas en una colonoscopia permiten establecer el diagnóstico en cerca del $60 \%$ de los casos, siempre y cuando se encuentren trofozoítos, para lo cual pueden requerirse coloraciones especiales (PAS) ya que la destrucción tisular puede ocultar su presencia $(6,7)$. La biopsia permite también distinguir entre carcinoma y adenoma, aunque, como en el caso presentado, puede mostrar también falsos negativos o confundir al clínico reportando un carcinoma inexistente.

El diagnóstico diferencial incluye obviamente carcinoma colorrectal, aunque también adenomas, tuberculosis intestinal, colitis ulcerativa, enfermedad de Crohn, absceso apendicular posapendicitis y abscesos por diverticulitis aguda (3).

Muchas veces no es posible establecer el diagnóstico de manera preoperatoria y es la pieza quirúrgica la que revela la presencia de los trofozoítos. La mortalidad en la época de la serie de casos más grande de amebomas, publicada en 1955, con datos de 78 casos del Instituto de Patología de las Fuerzas Armadas de los Estados Unidos, era hasta del $40 \%$ (10). En la actualidad, la mortalidad es mucho menor, aunque no ha sido establecida adecuadamente por la disminución notable en el número de casos.

Si se logra establecer el diagnóstico de manera endoscópica y preoperatoria, el tratamiento con metronidazol intravenoso seguirá siendo la primera elección tanto para los ataques agudos de colitis amebiana como para el absceso hepático y el ameboma. Este último puede incluso mejorar y desaparecer por completo después del tratamiento, el cual debe incluir también un antibiótico dada la necesidad de erradicar la infección bacteriana secundaria $(1,2)$.

En la actualidad, la cirugía debe reservarse para los casos que presenten complicaciones como obstrucción o perforación intestinal o cuando el diagnóstico no haya podido ser establecido con los métodos endoscópicos e histopatológicos (11).

La utilidad en nuestro medio de los test serológicos para amebiasis es controversial, ya que un gran porcentaje de la población los tendrá positivos. La medición de antígenos en materia fecal es otra buena alternativa cuando haya sospecha diagnóstica basada en los estudios endoscópicos e imagenológicos $(1,2)$.

Si el ameboma se presenta conjuntamente con un absceso hepático (12-14), el tratamiento de elección seguirá siendo el metronidazol intravenoso y el drenaje percutáneo del absceso hepático, cuando esté indicado. Actualmente es muy raro tener que llevar un absceso hepático a cirugía a menos que haya ruptura, se localice en el lóbulo hepático izquierdo y sea de gran tamaño o que esté localizado cerca del diafragma o del pericardio. Se desconoce la frecuencia de presentación simultánea de ruptura del absceso hepático y perforación del colon.

El caso clínico presentado asocia la presencia de un ameboma en el sitio quizá menos frecuente de los reportados en la literatura (como es el recto), un doble absceso hepático, el mal diagnóstico inicial con el lógico empeoramiento progresivo a pesar del tratamiento y la ruptura simultánea del ameboma y el absceso hepático. También enriquece la práctica clínica y permite enfatizar aún más en el altísimo índice de sospecha que se debe tener en casos similares en un país endémico como Colombia.

A pesar de no haber logrado una revisión de las placas y bloques de parafina de las biopsias tomadas en las dos primeras colonoscopias extrainstitucionales, se puede mencionar que la extensa necrosis, la gran respuesta inflamatoria, los cambios nucleares reactivos y la no visualización de trofozoítos llevaron a la confusión en el diagnóstico histológico, la cual también se presentó en la primera colonoscopia realizada en nuestro hospital y que se relaciona con las dificultades en el diagnóstico reportadas en la literatura. La pieza quirúrgica, tanto a nivel macro como microscópico, mostró los hallazgos clásicos del ameboma y el compromiso transmural por trofozoítos.

\section{REFERENCIAS}

1. Gómez J, Cortés J, Cuervo S, López M. Amebiasis intestinal. Infect 2007;11(1):36-45.

2. Haque R, Huston C, Hughes M. Amebiasis. N Engl J Med 2003;348:1565-73.

3. Majeed S, Ghazanfar A, Ashraf J. Caecal amoeboma simulating malignant neoplasia, ileocaecal tuberculosis and Crohn's disease. J Coll Physicians Surg Pak 2003;13:116-7.

4. Ng D, Kwok S, Cheng Y, Chung C, et al. Colonic amoebic abscess mimicking carcinoma of the colon. Hong Kong Med J 2006;12:71-3.

5. Simsek H, Elsurer R, Sokmensuer C. Amoeboma mimicking carcinoma of the cecum: case report. Gastrointest Endosc 2004;59:453-4. 
6. Mendell G, Benett J, Douglas R. Principles and practice of infectious diseases. 6th edition. New York: Churchill Livingstone; 2005.

7. Liu C, Crawford J. The gastrointestinal tract. In: Kumar V, Abbas AK, Fausto N, editors. Robbins and Cotran pathologic basis of disease. 7 th edition. Pennsylvania: WB Saunders Company; 2004. p. 839-40.

8. Misra S, Misra V, Dwivedi M. Ileocecal masses in patients with amebic liver abscess: etiology and management. World J Gastroenterol 2006;12:1933-6.

9. Rodea H, Athié C, Durán M, Montalvo E, et al. El comportamiento del ameboma en las últimas cuatro décadas. Experiencia en el Hospital General de México. Cir Gen $2008 ; 30(2)$.
10. Radke R. Ameboma of the intestine: an analysis of the disease as presented in 78 collected and 41 previously unreported cases. Ann Intern Med 1955;43(5):1048-66.

11. Radovanovic Z, Katic V, Nagorni A, Zivkovic V, et al. Clinical diagnostic problems associated with caecal amoeboma: Case report and review of the literature. Pathol Res Pract 2007;203:823-5.

12. Ray G, Iqbal N. Right colonic mass with hepatic lesion remember ameboma. Indian J Gastroenterol 2006;25:272.

13. Sharma D, Patel L, Vaidya V. Amoeboma of ascending colon with multiple liver abscesses. J Assoc Physicians India 2001;49:579-80.

14. Sachdev G, Dhol P. Colonic involvement in patients with amoebic liver abscess: Endoscopic findings. Gastrointest Endosc 1997;46:37-9. 